\title{
Parestesia do Nervo Alveolar Inferior associado à cirurgia de terceiro molar
}

\author{
Inferior Alveolar Nerve Paresthesia associated with third molar surgery
}

Recebido: 22/10/2021 | Revisado: 25/10/2021 | Aceito: 27/10/2021 | Publicado: 31/10/2021

\author{
Queren Hapuque Quezia dos Reis Bezerra \\ Universidade Brasil, Brasil \\ E-mail: querinhaareis@gmail.com \\ Débora Ellen Amorim de Souza \\ Universidade Brasil, Brasil \\ E-mail: deboraamorim_@hotmail.com \\ Lais Aragão Bandeira \\ Universidade Brasil, Brasil \\ E-mail: laisbandeira10@gmail.com \\ Thalitta Fiamma da Silva Bezerra \\ Universidade Brasil, Brasil \\ E-mail: Thalittafiih18@gmail.com \\ Paulo Roberto Gromatzky \\ Universidade Brasil, Brasil \\ E-mail: pgromatzky@gmail.com
}

\begin{abstract}
Resumo
A Parestesia do Nervo Alveolar Inferior tem como uma das causas, algum possível dano ao NAI ao realizar uma cirurgia de exodontia de terceiros molares inferiores, sendo essa uma das causas, abordar e discutir sobre este tema é de extrema importância para o conhecimento e menor ocorrência de lesão iatrogênica do Cirurgião Dentista ao NAI. A presente revisão de literatura visa abordar o conhecimento anatômicos do NAI em relação a cavidade oral e aos dentes que serão extraídos, fatores que podem ocasionar, como prevenir e a terapêutica caso ela ocorra.
\end{abstract}

Palavras-chave: Nervo alveolar inferior; Parestesias; Lesão; Terceiro molar inferior.

\begin{abstract}
Paresthesia of the Lower Alveolar Nerve has as a cause, some possible damage to the NAI when performing a surgery of lower third molars extraction, which is one of the causes, addressing and discussing this topic is extremely important for knowledge and less occurrence. of iatrogenic injury of the Dentist to NAI. This literature review aims to address the anatomical knowledge of the NAI in relation to the oral cavity and the teeth that will be extracted, factors that may cause, such as prevention and therapy if it occurs.
\end{abstract}

Keywords: Lower alveolar nerve; Paresthesias; Injury; Lower third molar.

\section{Introdução}

Os nervos cranianos são separados em pares, totalizando 12 pares sendo numerados por algarismos romanos, seguido por um nome específico que cada um deles possui. Estes 12 pares de nervos cranianos se dividem em 3 principais grupos: Sensitivos ou Sensoriais, Motores e Mistos. Eles estão ligados ao encéfalo onde ficam responsáveis pela resposta aos neurônios no córtex do cérebro e aos neurônios periféricos, onde exercem a função de sensação de dor, tato, temperatura e também a função de movimentação de alguns músculos responsáveis pela mastigação, expressões faciais e mímicas. (JOHANNES et al. 2006).

Dentre os nervos mistos está presente o quinto par V - Nervo Trigêmeo que ainda possui uma subdivisão em três ramos, Nervo Oftálmico, Nervo Maxilar e Nervo Mandibular. O ramo mandibular que a partir do gânglio trigeminal passa pelo forame oval saindo assim da parte craniana do esqueleto, se divide na fossa infra temporal em sete partes, Nervo massetérico, Nervo bucal, Nervo pterigóideo, Nervo temporal, Nervo aurículo-temporal, Nervo Lingual (NL) e Nervo Alveolar Inferior (NAI), sendo este último exclusivamente sensitivo. (JOHANNES et al. 2006). 
A ocorrência iatrogênica da parestesia resulta em alterações sensoriais que podem ser a todo tempo ou ocorrer em episódios anormais de dormência, formigamento, sensibilidade alterada ao frio ou calor e coceira, afetando a região do vermelho do lábio e no tecido do mento extraoral e intraoral a gengiva vestibular do primeiro pré-molar aos molares inferiores. O que pode ter duração de em média 6 meses ou ser permanente. (JULIANA 2016).

A proximidade anatômica do NAI com os terceiros molares inferiores (3MI) é uma das principais causas de Parestesia no pós-operatório, pois vem sendo realizadas com mais frequência cirurgias de exodontias, sendo assim necessário tomar todos os cuidados pré-operatórios com exames de imagem, como radiografia panorâmica e se possível uma tomografia computadorizada, diminuindo a chance de injúria ao NAI e sendo evitada pelo profissional cirurgião dentista, o qual deve ser capacitado para realização da cirurgia de exodontia de forma a trazer conforto e qualidade profissional ao paciente que pode ter uma mudança significativa na sua vida como dificuldade na fala, de se alimentares sendo alimentos sólidos ou líquidos, beijar, maquiar-se ou até mesmo fazer a barba. (GABRIELA et al. 2013).

Esse trabalho de revisão de literatura aborda em seu contexto a relação da cirurgia de exodontia de terceiros molares inferiores e a ocorrência iatrogênica de parestesia do NAI a fim de discutir as principais causas e efeitos que podem ser geradas, tratamentos que são utilizados caso ocorra e os métodos que podem auxiliar na prevenção da parestesia.

\section{Metodologia}

Por meio de uma revisão de literatura que foi abordado o tema abordado um estudo descritivo, cujo objetivo é compreender os conhecimentos e as condutas em relação Parestesia do Nervo Alveolar Inferior Associado a cirurgia de terceiro molares.

(KOCHE, 2011).

Os critérios de inclusão foram os seguintes: artigos e revistas, disponível nos idiomas em língua inglesa e portuguesa nos site como: Google Acadêmico, PubMed, Scielo (Scientific Eletronic Library Online) e BDTD (Biblioteca digital brasileira de tese e dissertações), contextos disponíveis em meios eletrônicos, que foram publicados entre1942 a 2021. Os critérios de exclusão: relatórios e publicações sem fontes confiáveis, materiais com disponibilidade incompleta que não abordava com clareza o tema.

\section{Revisão de Literatura}

\subsection{Anatomia das inervações da face}

Os nervos ligados ao encéfalo, também conhecidos como nervos cranianos são separados 12 pares. Eles são ligados ao córtex do cérebro por fibras chamadas corticonucleares, estas são formadas na protuberância anelar que fica situada no tronco cerebral. Neste tronco do encéfalo estão localizadas também 10 dos 12 núcleos que originam os nervos cranianos. (JOHANNES et al, 2006)

Classificando os 12 pares de nervos cranianos teremos 3 grupos, sendo eles: Sensitivo ou Sensoriais, Motores e Mistos. O grupo de nervos sensitivos ou sensoriais são destinados exclusivamente a sensibilidade, ou seja, estão relacionados apenas com os sintomas de sensibilidade em geral como dor, temperaturas, olfato, visão entre outros. (JOHANNES et al. 2006).

Neste grupo estão presentes:

- | - Nervo Olfatório

-\| - Nervo Óptico

- VIII - Nervo Vestibulococlear 
O grupo de nervos motores são os responsáveis pela movimentação dos músculos látero-posteriores do pescoço, músculos esqueléticos e movimentação dos olhos. (JOHANNES et al. 2006)

Neste grupo estão presentes:

- III - Nervo Oculomotor

- IV - Nervo Troclear

$\bullet$ VI - Nervo Abducente

-XI - Nervo Acessório

-XII - Nervo Hipoglosso

O grupo de nervos mistos é o grupo mais complexo dos 3 citados, pois nele há nervos sensitivos e motores e são responsáveis pela sensibilidade da pele, do couro cabeludo, além de serem responsáveis também pela movimentação dos músculos da face (expressões faciais), movimentação da mandíbula, posterior da língua e faringe além de fazer a enervação de glândulas parótidas e vísceras. (JOHANNES et al. 2006).

Neste grupo estão presentes:

$\bullet \mathrm{V}$ - Nervo Trigêmeo

- VII - Nervo Facial

- IX - Nervo Glossofaríngeo

-X - Nervo Vago

Através do ramo ascendente o NAI percorre o Canal Mandibular (CM) juntamente com a veia alveolar inferior e pela artéria alveolar inferior, realizando assim as inervações dos elementos dentários da mandíbula. (JOHANNES et al. 2006).

\subsection{Posicionamento dos terceiros molares em relação ao Nervo alveolar Inferior}

É de extrema importância analisar no pré-operatório a oposição que o dente em questão se encontra, para que seja escolhida a melhor forma de abordagem para a cirurgia proposta ao caso. Para estabelecer uma melhor comunicação entre os profissionais e realizar uma melhor avaliação, foram criadas diversas classificações, entre elas de George Winter em 1926, classificando os dentes quanto as suas angulações sendo elas: vertical, horizontal, distoangular e mesioangular. Enquanto Pell e Gregory em 1933, classificou em relação a profundidade de inclusão (classes A, B e C) e relação com o ramo mandibular (classes I, II e III), avaliações que podem ser feitas por exames de imagem auxiliares como panorâmica e tomografias que mostram na maioria dos casos nitidamente o canal mandibular e a posição do dentes em relação ao mesmo. (SANTOS et al. 2007). Terceiros molares inclusos mandibulares: incidência de suas inclinações, segundo classificação de Winter: levantamento radiográfico de 700 casos. (Revista Gaúcha de Odontologia, 2007).

A posição do dente parece es estar diretamente relacionada com lesões ao nervo alveolar inferior. Foi descrita a paralisia do nervo facial após remoção de terceiro molar inferior com resolução em sete dias, utilizando-se terapia com corticoides (BURKE et al. 1987).

\subsection{Classificação das lesões nervosas}

De acordo com Seddon, as lesões nervosas periféricas são classificadas em:

Neuropraxia - lesão leve com perda motora e sensitiva, sem alteração estrutural; (JONES R. 2010)

Axonotmese - é comumente vista em lesões por esmagamento, estiramento ou por percussão. Há perda de continuidade axonal e subsequente degeneração Walleriana do segmento distal. Nesse tipo de lesão não ocorre perda de célula 
de Schwann, e a recuperação irá depender do grau de desorganização do nervo e também da distância do órgão terminal. (SCARANO et al., 2007)

Neurotmese - separação completa do nervo, com desorganização do axônio causada por uma fibrose tecidual com consequente interrupção do crescimento axonal. A recuperação espontânea é pobre sem intervenção cirúrgica (Jerjes ET al. 2006)

Além da classificação de Seddon. Há outra conhecida pouco utilizada na prática, a classificação de Sunderland. Refinou a classificação de Seddon dividindo-a em cinco tipos ou graus. A neuropraxia foi classificada como tipo I; já a axonotmese foi dividida em três tipos, que variam de acordo com o grau da lesão, enquanto que a neurotmese foi classificada como tipo 5. É raramente possível, através da classificação de Sunderland, classificar com exatidão uma lesão axonotmética com base em dados clínicos e eletromiográficos. O subtipo é geralmente discriminável pelo exame histológico da lesão do nervo. (KIM et al. 2013.)

\subsection{Patofisiologia por três fenômenos principais}

3.4.1 Agressão mecânica: Estiramento: e o ato de exercer a força de tração quando ultrapassa a elasticidade do nervo; Laceração: é causada por instrumento afiados em contato direto com o nervo; Compressão: onde mantém a continuidade do nervo. (BOFFANO et al. 2012)

3.4.2 Agressão térmica: quando se utiliza instrumentos rotatórios sem ou com refrigeração insuficiente. (BOFFANO et al. 2012)

3.4.3 Agressão química: quando o nervo entra em contato com as (BOFFANO et al. 2012).

3.5 Possíveis fatores para ocasionar a parestesia. A idade avançada do paciente, a dificuldade operatória do caso e a proximidade entre o terceiro molar inferior e o canal mandibular. (LIU et al. 2015).

\subsubsection{Parestesia imediata:}

- Realizamos a incisão com o bisturi;

- Tensão exacerbada no afastamento do retalho;

- Na odontossecção ou osteotomia lesão pela broca;

- No ato de luxar e curetar o alvéolo, o pressionamento do nervo pelas raízes ou instrumento cirúrgico;

- Durante a sutura pela agulha.

3.5.2 Parestesias não-imediatas ou tardias, que se desenvolvem alguns dias após a intervenção cirúrgica:

- Compressão do nervo pelo edema pós cirúrgico ou coagulo sanguíneo;

- Coágulo com micro fragmentos ósseos resultantes da luxação que comprimem o nervo;

- Envolvimento do nervo pela reorganização fibrosa do coágulo, Barotrauma causado pela despressurização nas viagens de avião.

\subsection{Radiografia panorâmica}


Meio muito utilizado atualmente no planejamento das exodontias de 3M, para análise da proximidade com o canal mandibular $(\mathrm{CM})$ e as raízes dos dentes a serem extraídos, neste exame complementar é possível analisar os seguintes elementos que ajudam no diagnóstico mais preciso para uma cirurgia mais segura. (DÍAZ-TORRES et al. 1990)

- Escurecimento da raízes;

- Estreitamento das raízes;

- Curvatura das raízes- dilaceração;

- Ápices bífidos;

- Interrupção da cortical superior do CM.;

- Desvio do CM.

Estreitamento do CM (Classificação do tipo de sinal radiográfico de íntima relação com o canal mandibular conforme classificação de Félez-Gutiérrez et al. (1997), modificada por Gomes (2001).

\subsection{Tomografia Computadorizada (TC)}

Não é recomendado devido a exposição excessiva a radiação, sendo requerido quando na radiografia panorâmica existam dúvidas sobre a proximidade e posição do NAI em relação as raízes dos 3 molares, pois exibem a informação mais minuciosa e individualizada nos planos coronal, axial e sagital. (ROEDER et al. 2012)

As informações após a análise da TC como a relação vestibulo-lingual do 3 molar, as suas curvaturas, a quantidade de raízes, a perda da cortical do CM superior e a distância entre o NAI e o 3M ajudam o cirurgião dentista a realizar um planeamento da cirurgia mais detalhado, como: avaliar os locais de odontossecção/osteotomia de modo a evitar lesionar o NAI. (PEKER et al. 2014)

Segundo Drage (2002). Naqueles casos onde são necessárias imagens mais precisas e detalhadas ou quando a relação de proximidade é muito grande, a tomografia computadorizada está indicada, como o TC Scan, pois irá fornecer a localização exata do nervo alveolar inferior em relação às raízes dentárias. Entretanto, sua des vantagem é o custo elevado e a alta dose de radiação adquirida pelo paciente.

\subsection{Prognóstico de recuperação}

A parestesia do NAI em sua maioria não é definitiva e a sua recuperação varia entre 4 e 6 meses, e este prognóstico é bom, e se deve ao fato do nervo estar protegido em um canal ósseo na mandíbula, porém em alguns casos pode ser mais demorado, dando o fato de que dentro do CM pode-se prender espículas ósseas ou até fragmentos radiculares, que se não houver uma atenção maior pelo profissional em observar este ocorrido, pode levar a parestesia permanente. (NGUYEN et al. 2014)

Já a recuperação do NL é um pouco mais lenta, devido ao nervo estar localizado em meio a tecido mole e sua tendência são o de retrair e ser englobado por tecido cicatricial. (NGUYEN et al. 2014)

Cerca de 92-96\% dos casos de parestesia do NAI e em $87 \%$ em NL há uma boa recuperação da sensibilidade entre 4 e 8 meses após a cirurgia. Obtêm-se esses valores independentemente do sexo do paciente e fator de idade, entretanto em jovens apresenta uma variância entre valores ligeiramente mais favoráveis, já em pacientes mais velhos, superiores a 30 anos de idade, a permanência ou recuperação incompleta tende-se a ser mais frequentes. (NGUYEN et al. 2014)

\section{Tratamento}

\subsection{Tratamentos não cirúrgicos}

\subsubsection{Terapia a Laser}


A aplicação do laser de baixa intensidade na região afetada deve-se ser feita 3 vezes por semana em um período de 1 a 8 semanas. Com essa terapia a reparação do tecido traumatizado tende a acelerar a formação de novas fibras de colágeno e o desenvolvimento de uma revascularização, além de ajudar a redução do edema e processos inflamatórios. (LEUNG et al.2012)

\subsubsection{Acupuntura}

Um meio que pode ser útil para o tratamento e a recuperação da parestesia é a acupuntura, este método utiliza agulhas que podem ou não ser associados com estímulos elétricos, porém não existem tantos estudos que comprovem sua eficácia. Existem pontos específicos na região maxilofacial onde essas agulhas podem ser posicionadas, após 6 meses da finalização deste tratamento seus resultados começam a se manifestar, onde cerca de $81,3 \%$ mostram boa recuperação e melhoria significativa da sensibilidade nas lesões do NAI e aproximadamente 50\% nas lesões do NL. (LEUNG et al.2012)

\subsubsection{Tratamento crioterápico}

A utilização da crioterapia é feita extra oralmente aplicando gelo intensamente nas primeiras 24 horas após a suspeita de parestesia e após deve ser aplicado episodicamente durante uma semana. A utilização da crioterapia tem como foco a diminuição de possíveis edemas na face, pois com o edema instalado na face, podem ser causadas lesões secundarias. (RENTON et al. 2012).

\subsection{Tratamento Farmacêutico}

Existem fármacos que são utilizados comumente para a terapia de parestesias, em lesões agudas é preconizado a corticosteroides e de anti-inflamatórios não esteroides (AINEs), porém é de extrema importância por em conta a história médica do paciente antes de prescrever qualquer medicação. (RENTON et al. 2012).

\subsection{Tratamento cirúrgico}

Para a reparação do NAI e do NL existem algumas possibilidades, como a anastomose direta criada em ambas as extremidades, caso as terminações nervosas não possam ser aproximadas sem a necessidade de tencioná-las, (JONES et al. 2010), as opções cirurgias são:

- Neurólise externa do NAI e/ou NL;

- Reparação por sutura direta;

- Enxerto autógeno da veia safena ou veia facial;

- Enxerto autógeno do nervo sural da perna ou do nervo grande auricular do pescoço.

Após a lesão a degeneração do nervo afetado ocorre a partir de 4 a 6 meses, dado a este motivo a microcirurgia devese ocorrer nos primeiros meses. Na literatura cerca de 50\% dos casos apresentam melhora após a cirurgia, porém quando o paciente relata melhoria espontânea da região lesionada deve-se optar por uma intervenção não cirúrgica. (MISCH et al. 2010)

\subsection{Complicações no pós operatório}

A incidência de complicações após a extração do terceiro molar é baixa, como recessão gengival edema facial, dor pós-operatório, tomada seca, parestesia ou anestesia prolongada do nervo alveolar inferior e lingual. Onde os fatores de risco incluem idade, sexo, medicamento, tabagismo, periodontite, infecção anterior, higiene oral deficiente, e experiência do cirurgião, tempo de irrigação, número de dentes, técnica anestésicas inadequadas. 
Antes da extração do terceiro molar, o paciente deve ser informado sobre o tempo estimado da operação e outras complicações possíveis com base em evidências científicas e informações pré-operatórias para ajudar a fazer o plano cirúrgico. Um bom histórico médico e um bom planejamento auxiliam na operação e garantem um melhor tempo pós-operatório para o paciente.

\section{Discussão}

A frequência da exodontia dos terceiros molares faz com que as complicações no pós-operatório ocorram em maior número, pois a Parestesia também pode estar relacionada a falta de um bom planejamento cirúrgico ou até mesmo técnica cirúrgica utilizada pelo profissional.

A análise radiográfica consiste em um dos meios auxiliares para um bom planejamento cirúrgico, radiografia panorâmica é um exame bem utilizado para avaliação da posição do nervo alveolar inferior em relação aos terceiros molares. Mas nem sempre a panorâmica será o suficiente, podemos solicitar também uma tomografia computadorizada que fornece as imagens em três dimensões diferentes, levando em consideração que a panorâmica tem um custo menor do que a tomografia, e é mais fácil de ser avaliada.

Habilidade profissional tendo em um correto diagnóstico e utilização do instrumento adequado faz parte da melhor forma para prevenir a ocorrência da parestesia, ou seja, é de maior responsabilidade do profissional que está operando.

A parestesia ocorre geralmente devido aplicação inadequada de força, visualização deficiente ou mau uso de instrumentos durante a cirurgia. Não menos importante, devemos citar que o estresse e a ansiedade dos profissionais com menor experiência podem afetar o paciente.

Referente ao tratamento, para o retorno sensitivo do paciente (LEUNG et al. 2012) relata referente a aplicação do laser de baixa intensidade na região afetada sendo feita três vezes por semana em um período de 1 á 8 semanas buscando acelerar a formação de novas fibras de colágeno e o desenvolvimento de uma revascularização, controlando também o edema e evitando processos inflamatórios. O mesmo autor diz sobre acupuntura, método utilizado com agulhas (RENTON et al. 2012) contestou que a utilização de criou terapia é feita extra oralmente aplicando gelo intensivamente nas primeiras 24 horas após a suspeita da parestesia durante uma semana, evitando assim possíveis edemas na face. Misch et al (2010) acredita que no tratamento cirúrgico, para reparação do NAI E NL, pois existem algumas possibilidades juntando 2 vasos sanguíneos

\section{Conclusão}

Através da pesquisa realizada é possível chegar à conclusão de que existem formas para minimizar a chance de lesão ao NAI, porém que também há a possibilidade de lesão se não realizado corretamente e o cirurgião dentista deve ter ciência destes meios e realizar um pré-operatório minucioso alertado assim o(a) paciente sobre o risco e que há em cada caso, formas de tratamento e que estes podem resultar em sucesso, e que algumas vezes a lesão pode ser definitiva.

$\mathrm{Na}$ odontologia a lesão de parestesia é um dos fatores mais frequentes após procedimentos iatrogênicos, como por exemplo, a exodontia dos 3MI. Atualmente existem muitos meios de se conseguir recuperar a região afetada, com tratamentos invasivos e tratamentos conservadores.

Contudo essa perca de sensibilidade é geralmente passageira com duração de 3 à 6 meses, mas também há casos onde é permanente, porém os números destes são muito menores devido ao fato de que os $\mathrm{CD}$ possuem muitos meios de prevenir qualquer risco. Desta forma também se faz fundamental a utilização da técnica empregada com precisão, além da colaboração do paciente, tornando assim um estudo discutido pela comunidade cientifica. 


\section{Referências}

BOFFANO P., ROCCIA F. \& GALLESIO C. Lingual nerve deficit following mandibular third molar removal: review of the literature and medicolegal considerations. Oral Surg Oral Med Pathol Oral Radiol. 2012; 10-18.

BURKE R. H., ADAMS J. L. \& MISH A. A. Immediate cranial nerve paralysis during removal of a mandibular third molar. Oral Sur Oral Med Oral Pathol $1987 ; 63: 172-4$

CHIAPASCO M., DE CICCO L. \& MARRONE G. Efeitos colaterais e complicações associadas à cirurgia do terceiro molar. Oral Sug Oral Med Oral Pathol. 1993; 76: 412-20. https://doi.org/10.1016/0030-4220(93)90005-0

DRAGE N. A. \& RENTON T. Inferior alveolar nerve injury related to mandibular third molar surgery: An Unusual case presentation. Oral Surg Oral Med Oral Pathol. 2002; 93:358-361.

FÉLEZ-GUTIÉRREZ, J. Las lesiones del nervio dentario inferior en el tratamiento quirúrgico del tercer molar inferior retenido: aspectos radiológicos, prognósticos y preventivos. Archivos de Odontoestomatología, 1997; 13(2): 73-83.

FLOR L. C. DE S, TRINTA L. B, GOMES, A. V. S. F., FIGUEIREDO, R. B, SOUZA, A. C. A., SILVA, L. de C. N., GOMES, F. S., FREIRE, M. D. P., \& AGOSTINO, C. N. L. F. Fatores associados aos acidentes e complicações na extração de terceiros molares: uma revisão de literatura. RSD. agosto 2021;10(10):e281101018932

JERJES W., SWINSON B., MOLES DR., El-MAAYTA M., BANU B, \& UPILE T. Permanent sersory nerve impairment following third molar surgery: a prospective stud. Oral Surg Oral Med Oral Pathol Oral Radiol Endod. 2006; 1-7.

JOHANNES. Sobotta atlas de anatomia humana. 22. ed. Rio de Janeiro: Guanabara Koogan, 2006.

JONES R. Repair of the trigeminal nerve: a review. Australian Dental Journal. 2010; 112- 119.

JONES R. The use of vein grafts in the repair of the inferior alveolar nerve following surgery. Australian Dental Journal, $2010 ; 207-2013$.

KIM, J. E., SHIM, J. S., HUH, J. B., RIM, J. S., LEE, J. Y., \& SHIM, S. W. Altered sensation causes by periimplantitis: a case report. Oral Surg Oral Med Oral Pathol Oral Radiol. 2013; 9-13.

KOCHE, J. C. (2011). Fundamentos de metodologia científica.

LEUNG, Y. Y., FUNG, P. P. L., \& CHEUNG, L. K. Tratment modalities of neurosensory defict after lower third molar surgery: a systematic review. J Oral Maxilofac Surg, 2012; 768-778.

LIU, W., YIN, W., ZHANG, R., \& ZHENG, Y., LI, J. Diagnostic value of panoramic radiography in predicting inferior alveolar nerve injury after mandibular third molar extraction: a meta-analysis. Australian dental Association. 2015; 233-239.

MISCH, C. E., \& RESNIK, R. Mandibular nerve neurosensory impairment after dental implant surgery: management and protocol. Implant dentistry, 2010; $378-385$.

NGUYEN, E., GRUBOR, D., \& CHANDU, A. Risk factors for permanent injury of inferior alveolar and lingual nerves during third molar surgery.J Oral Maxilofac Surg, 2014; 2394-2401.

PEKER, I., SARIKIR, C., ALKURT, M. T., \& ZOR, Z. F. Panoramic radiography and cone-bean computed tomography findings in preoperative examination of impacted mandibular third molars. BMC Oral Health, 2014; 71.

PELL, G. J.; \& GREGORY, G.T. Report on a ten-year study of a tooth division technique for the removal of impacted teeth. American Journal of Orthodontics; 1942. 28(11): 660-666.

RENTON, T., YILMAN, Z., \& GABALLAH, K. Evaluation of trigeminal nerve injuries in relation to third molar surgery in a prospective patient cohort. Recommendations for prevention. Int. J. Oral Maxillofac Surg, 2012; 1509-1518.

ROEDER, F., WAHTLIN, D., \& SCHULZE, R. Necessity of 3D visualization for the removal of lower wisdom teeth: required sample size to prove noninferiority of panoramic radiography compared to CBCT. Clin Oral Invest. 2012; 699-706.

SANTOS-JÚNIOR, P. V. Terceiros molares inclusos mandibulares: incidência de suas inclinações, segundo classificação de Winter: levantamento radiográfico de 700 casos. Revista Gaúcha de Odontologia, 2007; 55(2): 143-147.

SCARANO, A., CARLO F., QUARANTA A., \& PIATTELI A. Injury of the inferior alveolar nerve after overfilling of the root canal with endodontic cement: a case report. Oral Surg Oral Med Pathol Oral Radiol Endod. 2007; 56-59. 